\title{
Hand-Shadow Illusions and 3D DDR Based on Efficient Model Retrieval
}

\author{
Jeng-Sheng Yeh ${ }^{* \dagger}$ Jen-Yuan Chiang ${ }^{* \dagger}$ Ting-Hao Huan ${ }^{\dagger}$ \\ Li-Fong $\operatorname{Lin}^{\dagger}$ \\ Ming-Yang $\mathrm{Yu}^{\dagger}$ \\ Jean Y.C. Tsai ${ }^{\dagger}$ \\ Chung-Lin Wen ${ }^{\dagger}$ Liang-Kai Wang ${ }^{\dagger}$ \\ Ding-Yun Chen ${ }^{\dagger}$ \\ Yung-Yu Chuang ${ }^{\dagger}$ \\ Bing-Yu Chen ${ }^{\dagger}$ \\ Ming Ouhyoung ${ }^{\#+}$ \\ National Taiwan University
}

\section{Introduction}

Just like the popular Dance Dance Revolution (DDR) dance game original created by Konami, we build an installation art system that can either let children play hand-shadow illusions, or let them pose their body within limited time with background music playing, so that the human pose can match the hint images dynamically shown on the screen.

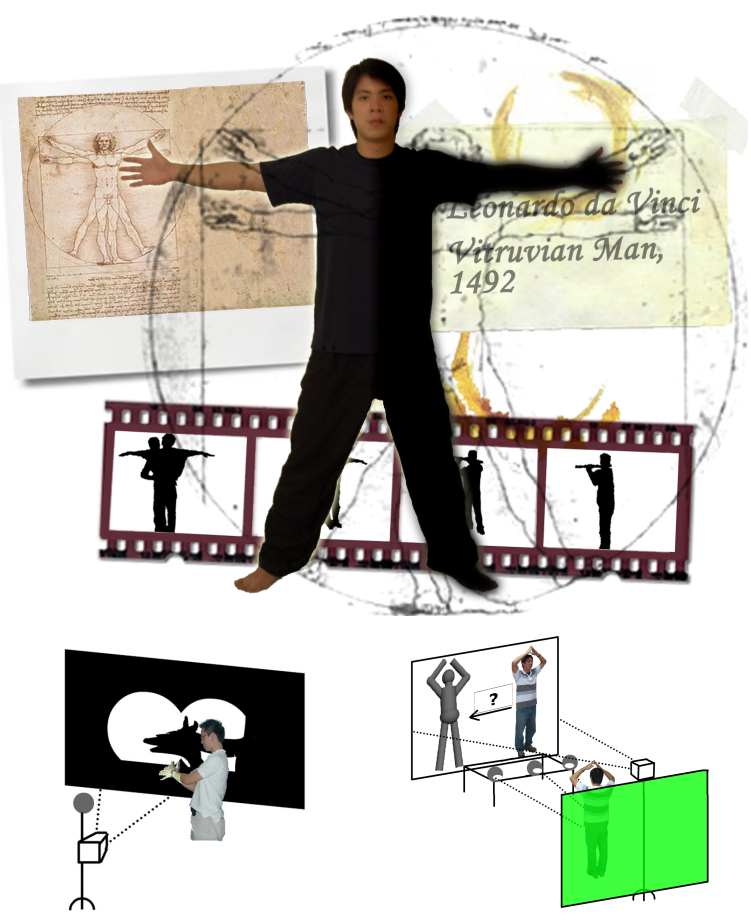

Figure 1: Playing in Hand-Shadow Illusions and 3D DDR with the help of a multi-view 3D model retrieval system in an efficient way.

\section{Exposition}

Two demos, Hand-Shadow Illusions and 3D DDR, share the same concepts and software engines, which are composed of three com-

\footnotetext{
*Joint first author

${ }^{\dagger}$ Email: $\{$ jsyeh, liubiti, richardg, lifong, yuminyung, jean, jonathan, yolux, dynamic, cyy, robin, ming $\} @$ cmlab.csie.ntu.edu.tw

$¥$ Contact person
}

ponents. The first component is a multi-camera system. Game players stand on a stage with several cameras surrounding them. Our capturing system uses background matting to segment the human body from the background and produces the silhouette images from many different views.

In Hand-Shadow Illusions, we use one camera to capture two different hand shadows at the same time on the same projector screen. It may also be possible to use two cameras in different places with network connection to play the game together. Furthermore, 3D DDR can use three cameras to capture different views at the same time.

The second component is an efficient model retrieval server. This server can receive a set of descriptors (dozens of integers) of each view as a query and find similar shapes from the model database. We transform the silhouette images obtained from the first component to a set of descriptors, submit those descriptors as query keys to this server, and get model candidates in terms of visual similarity.

Hand-Shadow Illusions uses a full-set of the database stored in the server, which has tens of thousands models. The return over HTTP is less than two seconds in average. On the other hand, 3D DDR uses a local database which contains only hundreds of models for real-time response.

The last component embeds those models in the display for the two demos.

\section{Conclusion}

We proposed an installation art system based on efficient model retrieval technology, and provided two demos, Hand-Shadow Illusions and 3D DDR.

\section{Acknowledgement}

We would like to thank Digimax and Ming Hsun Yu for providing the audios used in our system. This project is partially supported by NSC 94-2752-E002-007-PAE and NSC 94-2213-E-002-035.

\section{References}

Chen, D.-Y., Tian, X.-P., Shen, Y.-T., And Ouhyoung, M. 2003. On visual similarity based $3 \mathrm{~d}$ model retrieval. Computer Graphics Forum 22, 3, 223-232. (Eurographics 2003 Conference Proceedings).

Funkhouser, T., Min, P., Kazhdan, M., Chen, J., HalderMAN, A., DobKin, D., AND JACOBS, D. 2003. A search engine for 3d models. ACM Transactions on Graphics 22, 1, 83-105. 